\title{
Fatty acid synthase promotes breast cancer metastasis by mediating changes in fatty acid metabolism
}

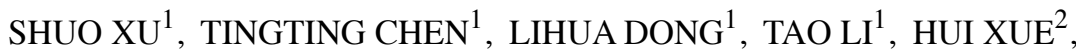 \\ BO GAO ${ }^{3}$, XIAODONG DING ${ }^{3}$, HUI WANG ${ }^{3}$ and HUA LI ${ }^{1}$ \\ ${ }^{1}$ West China School of Basic Medical Sciences and Forensic Medicine; \\ ${ }^{2}$ West China School of Medicine/West China Hospital, Sichuan University, Chengdu, Sichuan 610041; \\ ${ }^{3}$ Analytical and Testing Center, Sichuan University, Chengdu, Sichuan 610065, P.R. China
}

Received February 5, 2020; Accepted September 8, 2020

DOI: $10.3892 / \mathrm{ol} .2020 .12288$

\begin{abstract}
Fatty acid metabolism is closely associated with the occurrence and development of tumors. The aim of the present study was to investigate whether the key enzyme involved in fatty acid synthesis, fatty acid synthase (FASN), mediates fatty acid changes that affect the activity and migration of breast cancer cells, and whether specific fatty acids play a role in tumor metastasis. The difference in serum fatty acid profiles between patients with invasive ductal carcinoma (IDC) and healthy controls was evaluated by gas chromatography-mass spectrometry (GC-MS) fatty acid profile analysis, and it was revealed that five types of fatty acids may be potential tumor markers in IDC. Immunohistochemistry and GC-MS analysis revealed that FASN expression affected the serum fatty acid profiles of patients with IDC. Following FASN knockdown, the migration of SK-Br-3 breast cancer cells was inhibited, and the contents of various fatty acids both inside and outside the cell decreased, while the contents of various fatty acids inside and outside the cell increased following FASN overexpression. The results of the present study revealed that the expression level of FASN affected the content of fatty acids in IDC tissues and breast cancer cell lines, and that FASN-mediated changes in specific fatty acids promoted tumor cell migration.
\end{abstract}

\section{Introduction}

Cancer causes $\sim 13 \%$ of all deaths worldwide (1), with breast cancer being the most common type of cancer and the leading cause of cancer-associated mortality among women (2). Metastasis and drug resistance, two key obstacles to curing breast cancer, are associated with the metabolic activity

Correspondence to: Professor Hua Li, West China School of Basic Medical Sciences and Forensic Medicine, Sichuan University, 17 People's South Road, Chengdu, Sichuan 610041, P.R. China E-mail: lihua_scu@scu.edu.cn

Key words: fatty acids synthase, free fatty acids, invasive ductal carcinoma, breast cancer cells, metastasis of fatty acids. Compared with normal cells, tumor cells exhibit numerous substantial changes in metabolic activity, with changes in fatty acid metabolism being an important mechanism involved in epithelial-to-mesenchymal transition, invasion and metastasis of tumor cells (3). There are two ways for the body to obtain fatty acids: Exogenous dietary intake, and endogenous fatty acid synthesis within cells. Normal cells perform very little endogenous fatty acid synthesis, primarily relying on exogenous sources. In tumor cells and precancerous tissues, however, endogenous fatty acid synthesis may serve as a significant source of fatty acids driving the proliferation of tumor cells (4).

Fatty acid synthase (FASN) is a key enzyme involved in endogenous fatty acid synthesis. Mammalian FASN is a complex multifunctional enzyme that contains seven catalytic domains and a 4'-phosphopantetheine prosthetic group on a single 270-kDa polypeptide (5). Endogenous fatty acid synthesis not only maintains redox equilibrium and the relative levels of saturated and unsaturated fatty acids in tumor cells, but it is also involved in the formation of membrane lipids. In addition, fatty acid metabolites may further activate tumor cell signal transduction pathways (6). FASN expression is very low in the majority of normal cells, yet this enzyme is often upregulated in breast (7), oral (8), prostate (9), glioma (10) and ovarian cancer (11). Inhibition of FASN may decrease the proliferation and survival of tumor cells, and may further induce their apoptosis (12). FASN expression is also known to be closely associated with tumor grade and poor prognosis (13). The FASN inhibitor orlistat was shown to decrease the proliferation of orthotopic tongue squamous cell carcinoma cells (8). Similarly, breast cancer cells exhibited cell cycle arrest and decreased proliferation when the FASN signaling pathway was inhibited (14). FASN has also been demonstrated to play an important role in tumor invasion and metastasis, and the FASN inhibitor cerulenin or short hairpin RNA-mediated FASN knockdown decreased liver metastasis of colorectal cancer (15). However, to the best of our knowledge, whether FASN mediates changes in the levels of fatty acids that affect the proliferation and migration of breast cancer cells, and whether specific fatty acids play a role in tumor metastasis, has yet to be investigated. FASN expression has been demonstrated in different breast cancer cells (16). 
Among those, the expression level of FASN in human breast cancer SK-Br-3 cells was higher compared with that in other breast cancer cell lines, such as MCF-7 and MDA-MB-231. Therefore, in the present study, SK-Br-3 breast cancer cell lines were selected to investigate the effect of FASN on fatty acids in breast cancer cells.

In order to investigate whether FASN can affect the growth and migration of breast cancer cells by regulating fatty acid profiles, the present study investigated the difference in fatty acid profiles between patients with invasive ductal carcinoma (IDC) and healthy controls, and the association between FASN expression and serum fatty acids in patients with IDC. Furthermore, FASN was inhibited or overexpressed to verify the effect of its expression level on the fatty acid content of breast cancer cells.

\section{Materials and methods}

Cell lines and cultures. The human breast cancer cell line SK-Br-3 was purchased from the cell library of the Chinese Academy of Sciences. SK-Br-3 cells were cultured in DMEM (HyClone; Cytiva), supplemented with 10\% FBS (Natocor) under $5 \% \mathrm{CO}_{2} / 95 \%$ in a humidified incubator for $72 \mathrm{~h}$.

Serum sample collection. A total of 25 female patients with breast cancer who were hospitalized at the West China Hospital Affiliated with Sichuan University between December 2018 and April 2019 were included in the patient group for the present study (Table SI). The healthy controls included 19 women who underwent health examinations in the same hospital during the same time period. The TNM system was used to determine breast cancer stage in these patients. The inclusion criteria for patients with breast cancer were as follows: i) breast IDC confirmed by pathology, without distant metastasis. The exclusion criteria were as follows: i) non-IDC of the breast confirmed by pathology; ii) previous radiotherapy, chemotherapy or surgical treatment; iii) a family history of breast cancer; and iv) use of oral estrogen receptor (ER) drugs. For all patients, fasting venous blood was collected in the morning, and blood samples were allowed to naturally coagulate prior to centrifugation. Serum samples were collected and stored at $-80^{\circ} \mathrm{C}$. All patients and/or their families provided written informed consent prior to participation in the present study, and were informed in detail regarding the purpose and methods involved.

Immunohistochemistry (IHC). IHC was performed using the SPlink Detection kit (cat. no. SP9000; ZSGB-BIO; OriGene Technologies, Inc.), according to the manufacturer's protocol. The primary antibody used was mouse anti-human FASN (1:300; cat. no. sc-48357; Santa Cruz Biotechnology, Inc.). For the negative control, the primary antibody was replaced with PBS solution.

Analysis of the IHC staining results. A total of five random fields of view were assessed under a light microscope (Nikon ECLIPSE Ti-U; Nikon Corporation) at a magnification of $\mathrm{x} 400$. The staining intensity score and the proportion of FASN-positive cells were evaluated by a pathologist as follows: Staining intensity: i) 0 , negative; ii) 1 , weakly stained; iii) 2, moderately stained; and iv) 3, strongly stained. Staining extent (percentage of stained cells): i) 0 , none; ii) $1,1-20 \%$; iii) $2,21-40 \%$; iv) $3,41-60 \%$; v) $4,61-80 \%$; and vi) $5,81-100 \%$. The final immunoreactive score (IRS) of the FASN expression level was calculated by multiplying the staining intensity score with the staining extent score. IRS was dichotomised using X-tile software (version 3.4.7; Yale School of Medicine), which is a useful bioinformatics tool for outcome-based cut-point optimization. IRS $\leq 7.5$ was designated as low expression, while IRS $>7.5$ was designated as high expression (17).

$G C-M S$. Cell sample and serum sample preparation. Cells $\left(1 \times 10^{6}\right.$ per plate) in $10-\mathrm{cm}$ plates were incubated in growth media for 1 day, collected and washed with $0.9 \% \mathrm{NaCl}$. Lipids were extracted from the cells using procedures similar to the Folch method and evaporated under nitrogen gas, as previously described (18). After adding $0.75 \mathrm{ml}$ methanol/water solvent $(\mathrm{v} / \mathrm{v}=4: 1)$, the cells were harvested, pelleted and snap-frozen in liquid nitrogen. The serum sample was dissolved at room temperature. Methanol $(0.7 \mathrm{ml})$ and saturated heptadecanoic acid/methanol solution $(1 \mathrm{mg} / \mathrm{ml}, 20 \mu \mathrm{l})$ that served as an internal standard was added to the samples. The samples were suspended via vortex for $30 \mathrm{sec}$ and then left to stand for $10 \mathrm{~min}$. Following centrifugation at $13,000 \times \mathrm{g}$ at $4^{\circ} \mathrm{C}$ for $10 \mathrm{~min}$, the supernatant was collected. A total of $2 \mathrm{ml} \mathrm{5 \%} \mathrm{methanol} \mathrm{sulfate}$ solution was added, swirled for $60 \mathrm{sec}$, bathed at $62^{\circ} \mathrm{C}$ for $2 \mathrm{~h}$, cooled to room temperature, and centrifuged $(13,000 \mathrm{x} \mathrm{g} / \mathrm{min}$ at $4^{\circ} \mathrm{C}$ ) for $10 \mathrm{~min}$. The supernatant was placed in the sample bottle and $2 \mathrm{ml} \mathrm{n}$-hexane was added. After being swirled for $60 \mathrm{sec}$, the solution was stratified for $10 \mathrm{~min}$ and sampled from the n-hexane layer for testing.

GC-MS parameter setting. Metabolomics analysis was performed on a SHIMADZU instrument (Shimadzu Corporation). The experiments were performed following a previously described protocol (19). Raw data were obtained in a full scan mode. The samples were run at random, and blank samples and quality control samples were inserted during sample analysis.

Small interfering RNA (siRNA)-mediated gene knockdown. Cells were seeded in a 6 -well plate at a density of $6 \times 10^{5}$ cells per well. The FASN siRNA sequences were as follows: FASN siRNA\#1: 5'-GGAGCGTATCTGTGAGAAA-3'; and FASN siRNA\#2: 5'-CCGTGGACCTGATCATCAA-3'. The negative control siRNA sequences were as follows: 5'-TTCT CCGAACGTGTCACGTTT-3' (20). The Lipofectamine ${ }^{\mathrm{TM}}$ RNAi MAX reagent (Invitrogen; Thermo Fisher Scientific, Inc.) was incubated with $60 \mathrm{pmol}$ siRNA in OPTI-MEM (Gibco; Thermo Fisher Scientific, Inc.) for $5 \mathrm{~min}$ at room temperature. The siRNA-reagent mixture was then added to the culture media and incubated for $48 \mathrm{~h}$ prior to cell use in downstream experiments.

Plasmid construction. Stable SK-Br-3 cells overexpressing FASN containing rs17848945[G] were generated. In brief, cDNA encoding FASN was commercially synthesized (Youkang Science Instruments Co., Ltd.) and subcloned into the EcoRI and BamHI sites of the empty vector. SK-Br-3 cells 
were electroporated with empty vector and FASN expression vector. Positive colonies were then picked, expanded and verified by western blotting (21).

Wound healing assay. Cells $\left(5 \times 10^{5} / \mathrm{ml}\right)$ were plated in 6-well dishes and incubated overnight to yield confluent monolayers. On the following day, the cells were cultured in serum-free medium, and linear scratch wounds were made in the monolayer using a $10 \mu \mathrm{l}$ sterile pipette tip. Images were captured immediately $(0 \mathrm{~h})$ and subsequently at 24,48 and $72 \mathrm{~h}$ after wounding. At all time points, the width of the scratch area was measured at 4 points, allowing for a measurement of monolayer migration into the wounded area over time. The result was expressed as follows: Migration index $=(0 \mathrm{~h}$ scratch width $-\mathrm{x}$ h healing width)/0 h scratch width $\mathrm{x} 100$.

Western blotting. Proteins were extracted from cells using RIPA lysis buffer (Beyotime Institute of Biotechnology) containing PMSF (Amresco, LLC) at a dilution of 1:100. Protein concentrations were then measured via bicinchoninic acid protein assay kit (Beyotime Institute of Biotechnology). Proteins were next resuspended in sample loading buffer, boiled for $5 \mathrm{~min}$, and electrophoresed on a polyacrylamide $10 \%$ gel. The proteins were then transferred to a nitrocellulose membrane, which was blocked with $5 \%$ low-fat dried milk at room temperature for $1 \mathrm{~h}$. The blots were then probed with specific antibody against FASN (1:1,000; cat. no. sc-48357; Santa Cruz Biotechnology, Inc.) overnight at $4^{\circ} \mathrm{C}$, followed by rabbit-anti-mouse secondary antibody $(1: 2,000$; cat. no. sc-358914; Santa Cruz Biotechnology, Inc.) at room temperature for $2 \mathrm{~h}$. ECL chromogenic substrate (Beyotime Institute of Biotechnology) was then used for chemiluminescence imaging to measure protein levels.

Transwell cell migration assay. Cells were initially serumstarved for $12 \mathrm{~h}$, and were then harvested and resuspended in serum-free media at $5 \times 10^{5}$ cells $/ \mathrm{ml}$. The cells were then plated using a 24-well Transwell chambers, with $200 \mu$ l cell suspension added to the upper chamber and $700 \mu \mathrm{l}$ culture medium containing 20\% FBS added to the lower chamber. After $48 \mathrm{~h}$, the Transwell chamber was removed, the original medium was discarded, and cells were washed twice with PBS, after which time the lower chamber was fixed with $100 \%$ methanol at room temperature for $30 \mathrm{~min}$. The chambers were then air-dried and stained with $0.1 \%$ crystal violet solution on a shaking table for $20 \mathrm{~min}$ at room temperature. The wells were then washed twice with PBS; cells that did not migrate were gently removed, and the samples were allowed to dry at $37^{\circ} \mathrm{C}$. The stained Transwell chamber was placed under an optical microscope (magnification, x100), and the cells were counted in five randomly selected fields.

Statistical analysis. All data are presented as mean \pm SEM. GraphPad Prism (version 8.0; GraphPad Software, Inc.) was used for all statistical analyses. Group sizes per experiment were based on a power analysis. Unpaired Student's t-test or one-way ANOVA were used as appropriate to determine statistical significance. Bonferroni's post hoc test was used where appropriate. $\mathrm{P}<0.05$ was considered to indicate a statistically significant difference.

\section{Results}

Differences in serum fatty acid profile between patients with IDC and healthy controls. An increase in fatty acid synthesis is one of the most obvious metabolic changes in tumor cells. The final products of fatty acid metabolism form signaling molecules that mediate the activation of tumor signal transduction pathways, thereby promoting tumor growth, angiogenesis, survival and metastasis (22). In order to investigate which fatty acids affect tumor metastasis and invasion, the present study assessed serum free fatty acids (FFAs) in 25 patients with IDC and 19 healthy controls. It was revealed that the levels of 12 fatty acids were significantly increased in the serum of patients with IDC, including 6 saturated fatty acids (C14:0, C15:0, C16:0, C18:0, C20:0 and C22:0), 2 monounsaturated fatty acids (C18:1 and C20:1), and 4 polyunsaturated fatty acids (C18:2, C20:4, C22:4 and C22:6) (Fig. 1A and B and Table SII). According to the analysis of GC-MS metabolites in IDC patients and healthy controls, 17 metabolites were identified via a National Institute of Standards and Technology (NIST) library search. The loading graph of Orthogonal Projections to Latent Structures Discriminant Analysis (OPLS-DA) model output demonstrated that $\mathrm{C} 18: 1, \mathrm{C} 18: 2, \mathrm{C} 16: 0, \mathrm{C} 20: 4$ and C18:0 were furthest from the origin, and may represent potential tumor markers (Fig. 1C). The potential tumor markers in patients with IDC were obtained from the results of metabolite extraction with Variable Importance in Projection and the results of the loading graph. The results revealed that C18:1, C18:2, C16:0, C20:4 and C18:0 may be potential tumor markers for IDC (Fig. 1C and D). These data suggest that multiple fatty acids may be involved in the metastasis and invasion of breast cancer.

Association between FASN expression and serum fatty acid metabolism in patients with IDC. FASN is an important biological synthetase and a key enzyme of tumor lipid production. The present study investigated the association between FASN expression and fatty acid metabolism in patients with IDC. Our previous studies demonstrated an increased expression level of FASN in lymph node metastases, IDC clinical stages II and III, and larger tumors ( $>2 \mathrm{~cm})$ (23). This means that FASN is involved in tumor growth and metastasis. FASN expression in 25 patients with IDC was next assessed by IHC staining. There were 12 cases in the high expression group and 13 cases in the low expression group (Fig. 2A and Table I). The levels of different FFAs were then compared between patients with high and low FASN expression levels, and the levels of 4 fatty acids were found to be significantly increased in patients with IDC with high FASN expression compared with those with low expression. These included two saturated fatty acids (C16:0 and C18:0), one unsaturated fatty acid (C18:1) and one polyunsaturated fatty acid (C20:4) (Fig. 2B and C and Table SIII). These data suggested that the expression of FASN may affect the FFA profiles in the serum of patients with IDC, and that FASN may be involved in tumor proliferation and migration, which is mediated through changes in fatty acid levels.

Effect of FASN on fatty acidmetabolism in breast cancer cells. FASN is upregulated in a variety of malignant tumors and is 

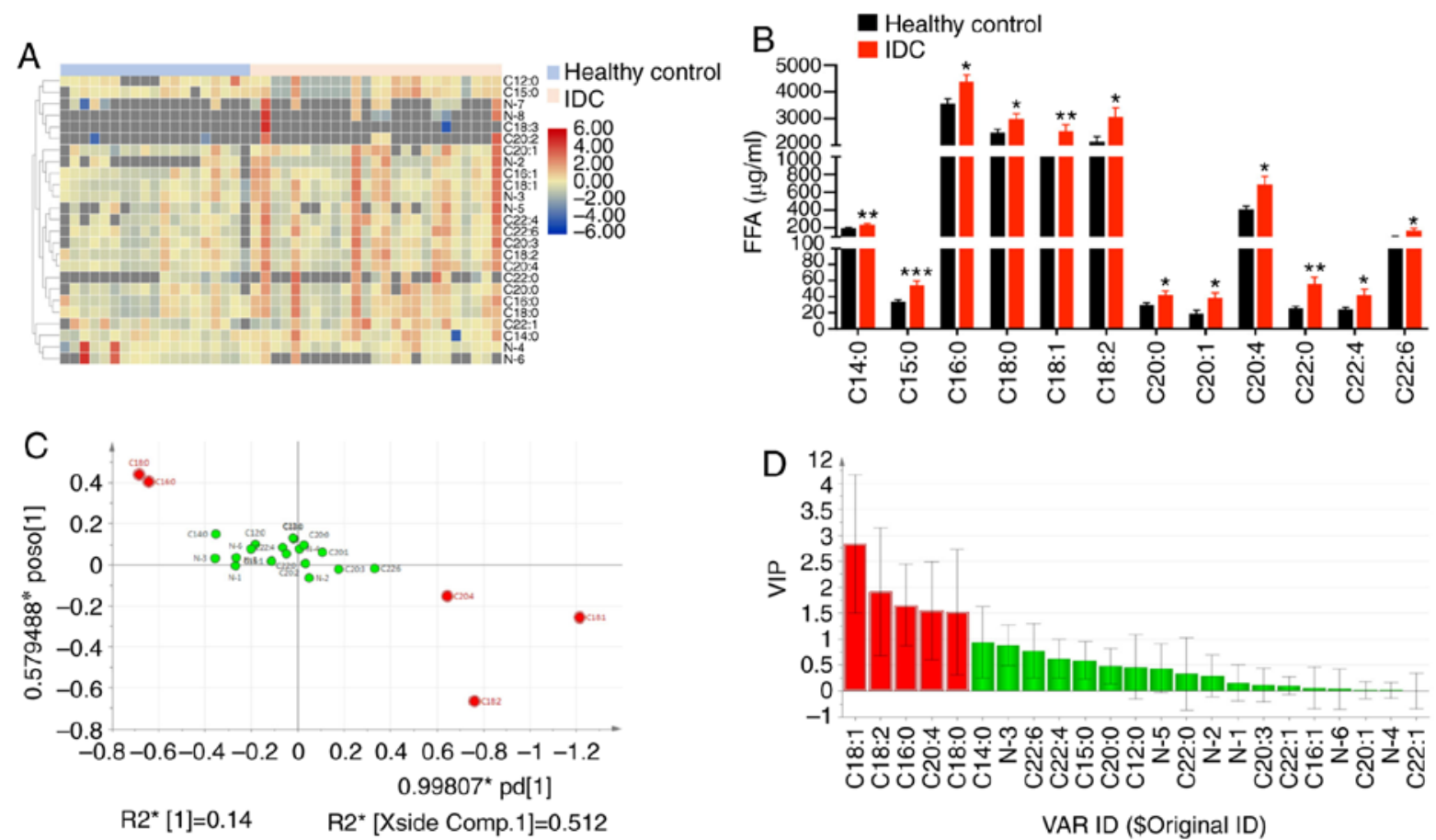

Figure 1. Serum fatty acids differed between IDC patients and healthy controls. (A) Heatmap showing the representation of serum FFAs in 19 healthy controls and 25 IDC patients. (B) The content of various fatty acids in the serum of IDC patients was significantly increased. Columns, mean ( $\mathrm{n}=19,25)$; bars \pm SEM, ${ }^{*} \mathrm{P}<0.05,{ }^{* * *} \mathrm{P}<0.01,{ }^{* * * *} \mathrm{P}<0.001$ vs. control. (C) OPLS-DA loading plot of IDC patient and healthy control groups. (D) VIP value of FFA spectrum between IDC patient and healthy controls, Red column, VIP value $>1$. IDC, invasive ductal carcinoma; FFAs, free fatty acids; VIP, variable importance of projection; OPLS-DA, orthogonal projections to latent structures discriminant analysis.

associated with tumorigenesis and metastasis. However, to the best of our knowledge, the effect of FASN-mediated fatty acid changes on tumor cell proliferation and migration has not yet been reported. To investigate whether FASN affects cell proliferation and metastasis by regulating fatty acid metabolism in breast cancer cells, SK-Br-3 cells expressing FASN were selected to inhibit and overexpress FASN in order to detect whether changes in fatty acid metabolism occurred in intracellular and extracellular media. Western blotting revealed that the FASN protein levels were significantly decreased in SK-Br-3 cells following treatment with $20 \mu \mathrm{g} / \mathrm{ml}$ of the FASN inhibitor cerulenin for $24 \mathrm{~h}$. The two different FASN-specific siRNA constructs also significantly inhibited FASN expression. Silencing was more efficient for the siRNA\#1 construct; thus, this siRNA was used in subsequent experiments. SK-Br-3 cells with FASN overexpression were constructed (Fig. 3A-D). In SK-Br-3 cells, the FASN was knocked down and overexpressed. The content and composition of FFAs in the intracellular and extracellular medium were determined by GC-MS. It was revealed that the composition of extracellular fatty acid was lower compared with that of intracellular fatty acids (Fig. 3E and I). The expression of FASN was decreased or upregulated, and the composition of FFA remained unchanged, but the fatty acid content was altered. After FASN was decreased by cerulenin $(20 \mu \mathrm{g} / \mathrm{ml})$, the levels of 5 fatty acids, including C14:0, C16:1, C18:1, $\mathrm{C} 18: 0$ and $\mathrm{C} 22: 4$, were decreased in the cells (Fig. 3E and F). After FASN was silenced by siRNA, the levels of 7 fatty acids, including C12:0, C14:0, C16:1, C16:0, C18:1, C18:0 and $\mathrm{C} 22: 4$, were decreased in the cells (Fig. 3E and G). Following overexpression of FASN, the levels of 8 fatty acids, including
C16:1, C16:0, C18:2, C18:1, C18:0, C20:0, C22:4 and C22:0, were increased in the cells (Fig. 3E and H). After FASN was decreased by cerulenin and siRNA, the levels of 4 fatty acids, including C12:0, C14:0, C16:0 and C18:0, were decreased in the medium (Fig. 3I and J). After FASN was overexpressed, the levels of 3 fatty acids, including C14:0, C16:0 and C18:0, were increased in the medium (Fig. 3I and K). After FASN expression was decreased, the levels of FASN-regulated fatty acids inside and outside the cell were decreased; similarly, after FASN expression was increased, the levels of FASN-regulated fatty acids inside and outside the cell were increased. Therefore, FASN may promote the proliferation and migration of cancer cells by altering the metabolism of fatty acids.

Decreased FASN expression significantly inhibits the migration of SK-Br-3 cells. The present study investigated the effect of FASN on the migration ability of breast cancer cells. A wound healing assay revealed that the migration of SK-Br-3 cells in the FASN group was inhibited by cerulenin and FASN siRNA (Fig. 4A-D), and was significantly decreased when compared with that in the controls. The Transwell experiment revealed that, after $48 \mathrm{~h}$ of treatment of SK-Br-3 cells with cerulenin or FASN siRNA (Fig. 4E-H), migration was significantly decreased compared with the controls, which was consistent with the results of the wound healing assay. In conclusion, when FASN in SK-BR-3 cells is decreased, cell migration is inhibited. Combined with the changes in fatty acids after FASN was decreased or increased, the findings suggest that FASN may promote tumor development by mediating changes in the levels of specific fatty acids. 
A
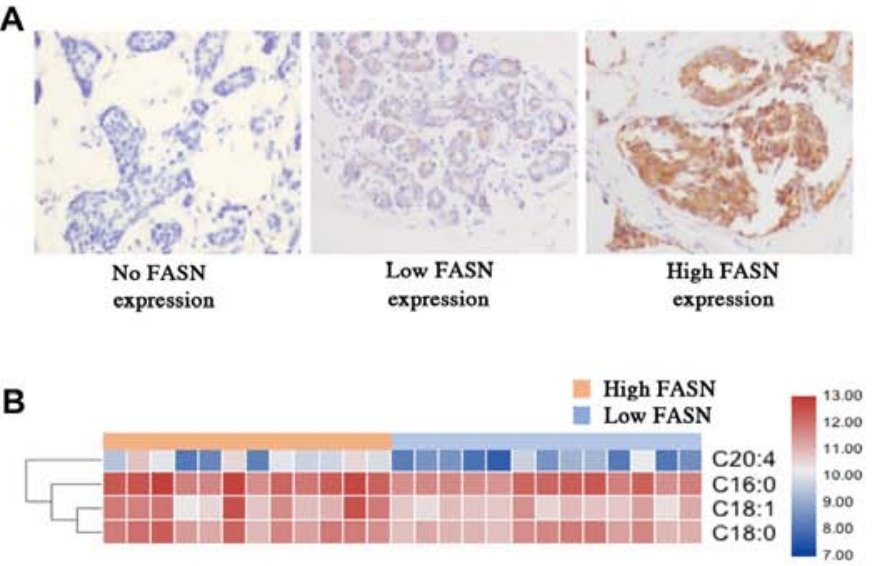

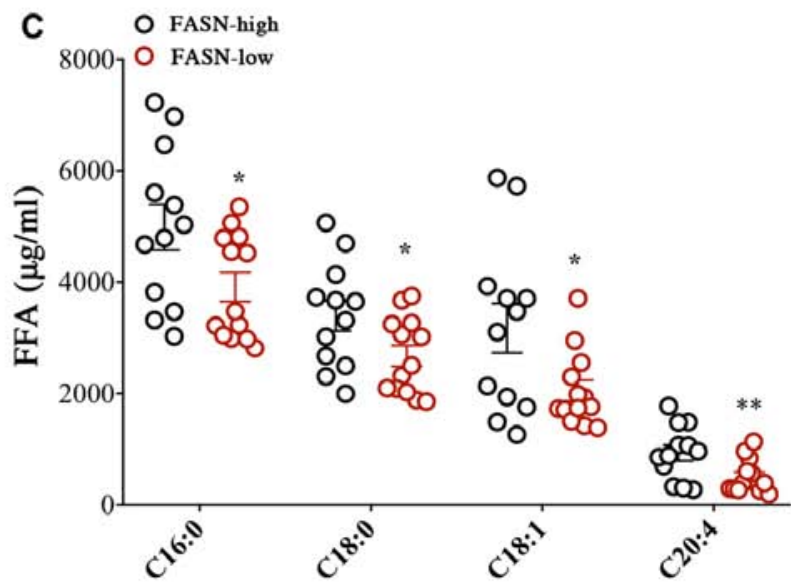

Figure 2. The expression of FASN affects the FFA content in the serum of IDC patients. (A) Immunohistochemistry staining showing FASN expression in IDC patient tissues; magnification, x400. (B) Heatmap showing the representation of serum FFAs in 12 patients with high and 13 patients with low FASN expression. (C) The levels of four FFAs were found to be significantly higher in IDC patients with high FASN expression compared with those with low FASN expression. Bars represent the means \pm SEM ( $\mathrm{n}=12$ and 13 , respectively); ${ }^{*} \mathrm{P}<0.05,{ }^{* *} \mathrm{P}<0.01$ vs. control. IDC, invasive ductal carcinoma; FFAs, free fatty acids; FASN, fatty acid synthase.

Table I. Association between FASN expression and clinicopathological parameters in IDC.

\begin{tabular}{|c|c|c|c|}
\hline \multirow[b]{2}{*}{ Parameters } & \multicolumn{3}{|c|}{ FASN expression, n (\%) } \\
\hline & High & Low & No. \\
\hline \multicolumn{4}{|l|}{ Age, years } \\
\hline$\leq 50$ & $8(32)$ & $10(40)$ & 18 \\
\hline$>50$ & $4(16)$ & $3(12)$ & 7 \\
\hline \multicolumn{4}{|c|}{ Lymph node metastasis } \\
\hline Negative & $6(24)$ & $12(48)$ & 18 \\
\hline Positive & $6(24)$ & $1(4)$ & 7 \\
\hline \multicolumn{4}{|l|}{ IDC stage } \\
\hline $0-1$ & $0(0)$ & $5(20)$ & 5 \\
\hline 2 & $4(16)$ & $1(4)$ & 5 \\
\hline 3 & $8(32)$ & $7(28)$ & 15 \\
\hline \multicolumn{4}{|c|}{ IDC size, $\mathrm{cm}$} \\
\hline$\leq 2$ & $5(20)$ & $11(44)$ & 16 \\
\hline$>2$ & $7(28)$ & $2(8)$ & 9 \\
\hline \multicolumn{4}{|l|}{ ER } \\
\hline Negative & $5(20)$ & $1(32)$ & 6 \\
\hline Positive & 7 (28) & $12(32)$ & 19 \\
\hline \multicolumn{4}{|l|}{ PR } \\
\hline Negative & $4(16)$ & $3(12)$ & 7 \\
\hline Positive & $8(32)$ & $10(40)$ & 18 \\
\hline
\end{tabular}

IDC, invasive ductal carcinoma; ER, estrogen receptor; PR, progesterone receptor; FASN, fatty acid synthase.

\section{Discussion}

The present study analyzed FFA levels in the serum of patients with IDC and healthy controls, and revealed that the serum FFA levels were significantly increased in patients with IDC.
In total, 12 fatty acids were found to be significantly increased, including 6 saturated fatty acids (C14:0, C15:0, C16:0, C18:0, C20:0 and C22:0), 2 monounsaturated fatty acids (C18:1 and $\mathrm{C} 20: 1)$ and 4 polyunsaturated fatty acids (C18:2, C20:4, $\mathrm{C} 22: 4$ and C22:6). Previous studies confirmed that the levels of various fatty acids in the plasma of patients with breast cancer, including C14:0, C16:0, C16:1, C18:0, C18:1, C18:2, C20:1 and C20:4, were higher compared with those in healthy controls (24). The levels of C16:0, C18:0 and C18:2 and total FFA in the serum of patients with IDC have also been found to be higher compared with those in normal controls (25), similar to the findings of the present study. The levels of the polyunsaturated arachidonic acid (C20:4) (26) and the saturated palmitic acid (C16:0) (27) were reported to be higher in breast cancer tissue compared with those in normal breast tissue, which may be used for distinguishing between these two tissue types. The present study also revealed that the levels of palmitic acid (C16:0) and arachidonic acid (C20:4) were upregulated in the serum of patients with IDC. In addition, the effects of $\omega-3$ Series docosahexaenoic acid (DHA C22:6), $\omega-6$ series linoleic acid (LA C18:2), arachidonic acid (AA C20:4) and $\omega-9$ series oleic acid (AO C18:1) on the differentiation, proliferation and metastasis of tumor cells have been previously reported. Both in vitro and in vivo experiments demonstrated that LA $(\mathrm{C} 18: 2)$ and $\mathrm{AO}(\mathrm{C} 18: 1)$ promoted the growth, metastasis and invasion of breast cancer cells. AA (C20:4) promotes breast cancer cell migration and invasion through the PI3K/Akt-dependent pathway (28). We herein detected that the levels of AA (C20:4), AO (C18:1) and LA (C18:2) were upregulated in IDC serum, and the levels of AA and $\mathrm{AO}$ in breast tissues with high FASN expression were higher compared with those in tissues with low FASN expression. However, it was previously reported that DHA (C22:6) inhibited the growth of breast cancer cells, but we detected that the content of DHA (C22:6) was increased in the serum of patients with IDC. It may be hypothesized that this increase in DHA (C22:6) may be caused by breast cancer metastasis. Other studies have found that the C12:0 and C14:0 levels were 

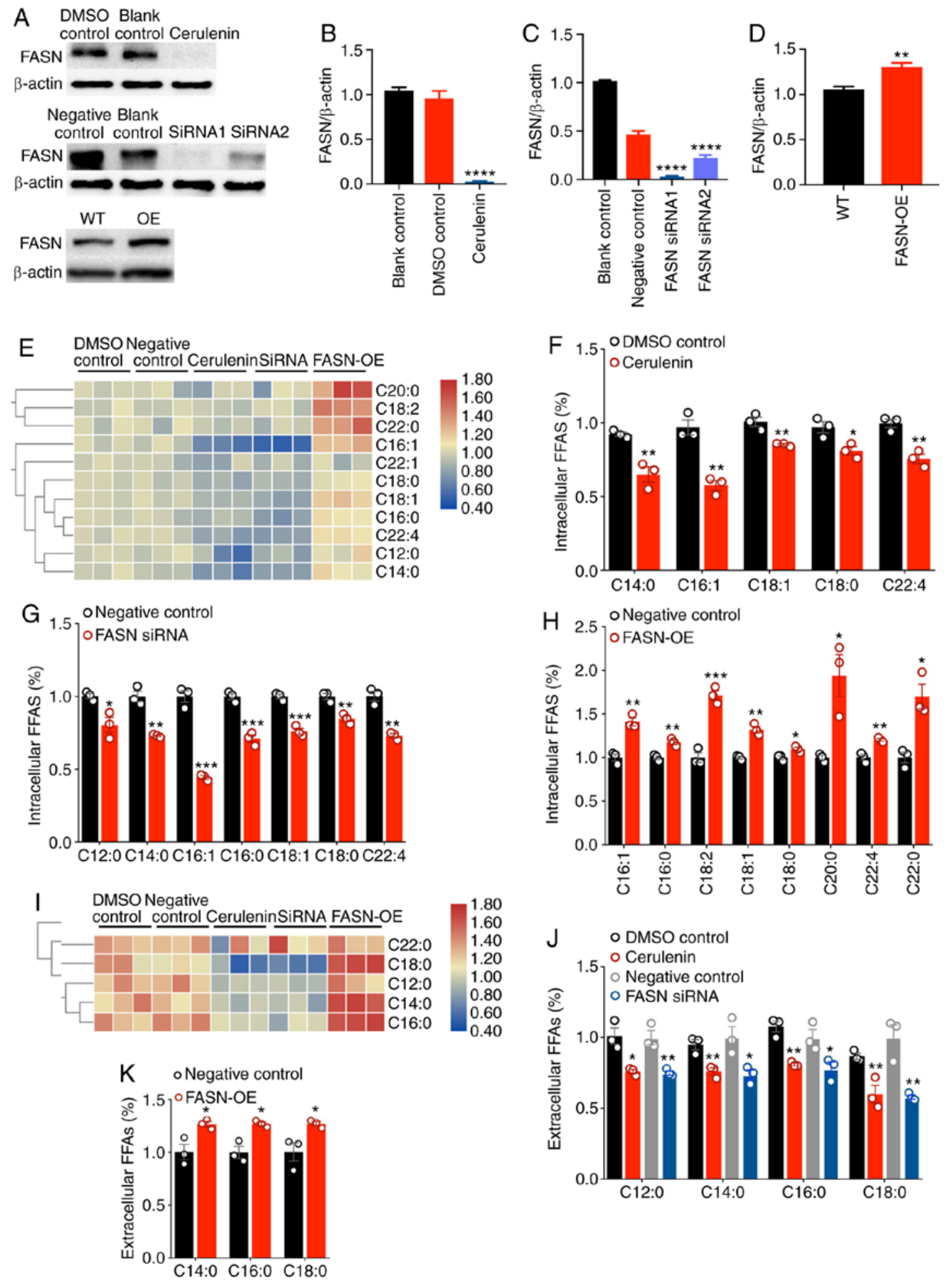

Figure 3. The expression level of FASN affects the fatty acid content of breast cancer cells. (A-D) FASN expression was significantly decreased following cerulenin and siRNA treatment; in addition, FASN was overexpressed in SK-Br-3 cells (FASN-OE SK-Br-3 cells). Bars represent the means \pm SEM ( $n=3$ ); ${ }^{* *} \mathrm{P}<0.01$, ${ }^{* * * * *} \mathrm{P}<0.0001$ vs. control. (E) Heatmap showing the changes in fatty acids in cells following FASN knockdown and overexpression. (F) FASN was inhibited by cerulenin, and the content of 5 fatty acids in cells was decreased; columns, mean ( $\mathrm{n}=3$ ); bars, $\pm \mathrm{SEM}$; ${ }^{*} \mathrm{P}<0.05,{ }^{* *} \mathrm{P}<0.01$ vs. DMSO control. (G) After FASN was silenced by siRNA, the content of 7 fatty acids in cells was decreased; columns, mean ( $\mathrm{n}=3)$; bars, $\pm \mathrm{SEM}$; ${ }^{*} \mathrm{P}<0.05,{ }^{* *} \mathrm{P}<0.01,{ }^{* * * *} \mathrm{P}<0.001$ vs. negative control. (H) After FASN was overexpressed, the content of 8 fatty acids in cells was increased; columns, mean ( $\mathrm{n}=3)$; bars, \pm SEM; ${ }^{\prime \prime} \mathrm{P}<0.05,{ }^{* * *} \mathrm{P}<0.01,{ }^{* * *} \mathrm{P}<0.001$ vs. negative control. (I) Heatmap showing the changes in extracellular fatty acids following FASN knockdown and overexpression. (J) FASN was inhibited by cerulenin and siRNA, and the content of 4 FFAs in the cell medium was reduced; columns, mean ( $\mathrm{n}=3)$; bars, $\pm \mathrm{SEM}$; ${ }^{*} \mathrm{P}<0.05$, ${ }^{* * *} \mathrm{P}<0.01 \mathrm{vs}$. control. (K) After FASN was overexpressed, the content of 3 types of FFAs in the cell medium was increased; columns, mean ( $\mathrm{n}=3$ ); bars, \pm SEM; " $\mathrm{P}<0.05$ vs. negative control. FASN, fatty acid synthase; FFAs, free fatty acids.

lower in highly metastatic T47D cells in the fusion state when compared with those in poorly metastatic breast cancer T47D cells in a sparse state (18). The C12:0, C14:0 and C18:0 levels in breast cancer adipose tissue were also lower compared with 


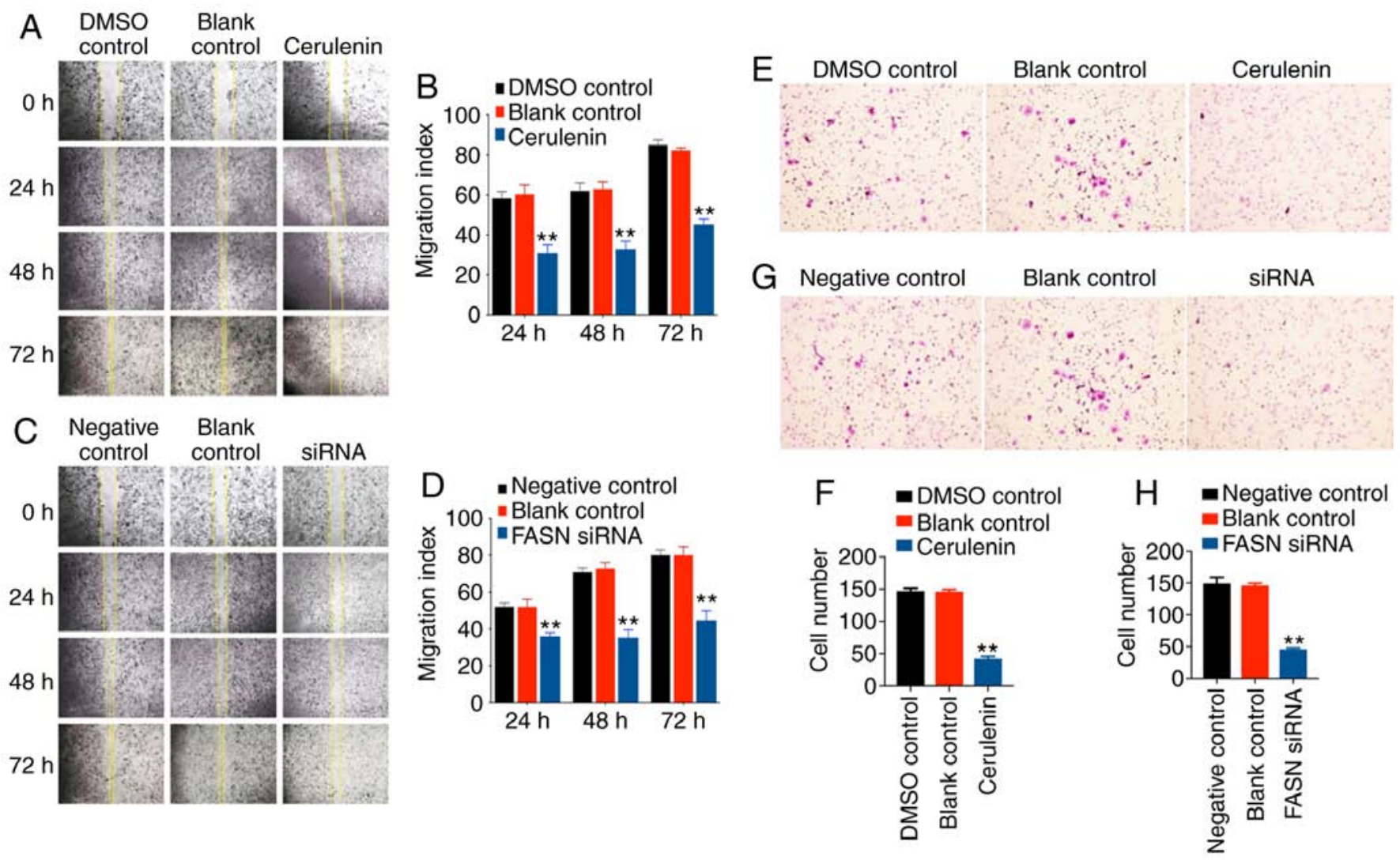

Figure 4. SK-Br-3 cell migration was significantly decreased following cerulenin and siRNA treatment. (A and B) Wound healing assay was performed to determine the effects of cerulenin $(20 \mu \mathrm{g} / \mathrm{ml})$ on SK-Br-3 cell migration. Columns and bars represent means $\pm \mathrm{SEM}(\mathrm{n}=3) ;{ }^{* *} \mathrm{P}<0.01$ vs. control. (C and D) Wound healing assay was performed to determine the effects of siRNA on SK-Br-3 cell migration. Columns and bars represent means \pm SEM $(n=3) ;{ }^{* *} \mathrm{P}<0.01 \mathrm{vs}$. control. (E and F) Images of Transwell migration assays revealing that cerulenin $(20 \mu \mathrm{g} / \mathrm{ml})$ suppressed SK-Br-3 cell migration. Columns and bars represent means $\pm \mathrm{SEM}(\mathrm{n}=3) ;{ }^{* *} \mathrm{P}<0.01$ vs. control. ( $\mathrm{G}$ and $\left.\mathrm{H}\right)$ Images of Transwell migration assays revealing that siRNA suppressed SK-Br-3 cell migration. Columns and bars represent means $\pm \mathrm{SEM}(\mathrm{n}=3) ;{ }^{* *} \mathrm{P}<0.01$ vs. control. FASN, fatty acid synthase.

those in the adipose tissue of benign breast conditions (28). These results were slightly different from the results of the present study, potentially due to different sources of experimental materials, genetic backgrounds of the participants and research methods used. In addition, the 25 patients with IDC in the present study were divided into high and low FASN expression groups by IHC. It was revealed that the levels of C16:0, C18:0, C18:1 and C20:4 in patients with IDC with high FASN expression levels were significantly higher compared with those with low FASN expression levels. Therefore, C16:0, C18:0, C18:1 and C20:4 may be potential tumor markers. The expression of FASN affected the serum content of fatty acids in patients with IDC, and this suggested that FASN-regulated fatty acids may be involved in tumor growth and migration.

It was previously demonstrated that palmitic acid (C16:0), a major FASN metabolite, can enhance the invasiveness of pancreatic cancer cells (29). When tumor cells shift from a proliferative to a migratory state, the absorption of fatty acids or the selective release of specific fatty acids from membrane lipids may help in the formation of signaling molecules that promote cell migration and invasion (30). It was previously reported that, in highly invasive and ER-negative breast cancer tissues, primary or secondary FASN metabolites (including C14:0, C16:0 and C18:0) may be associated with poor patient prognosis (31). Therefore, tumor cells may render themselves less sensitive to oxidative stress via increasing the saturated fatty acid content of cell membranes, thereby improving tumor cell survival (32). Therefore, FASN can mediate the generation of lauric acid (C12:0), myristic acid (C14:0), palmitic acid (C16:0) and stearic acid (C18:0), which are signaling molecules involved in lipid signal transduction, and play an important role in the survival and migration of tumor cells. The present study demonstrated that, after inhibiting FASN, the content of intracellular and extracellular FFAs decreased, and cell activity and migration were inhibited. Following FASN overexpresion, the content of FFAs inside and outside the cells increased. FASN has also been previously shown to affect the activity and migration of multiple cancer cells (33). This may be due to the fact that FASN increases endogenous fatty acid synthesis in order to meet the energy demands associated with the rapid proliferation and differentiation of tumor cells and to provide substrate material for membrane lipid synthesis. Fatty acid synthesis metabolites may serve as signaling molecules necessary for tumor development and progression. Therefore, FASN-mediated fatty acid synthesis metabolites warrant further investigation, as they may represent important signaling molecules that regulate gene expression and cell phenotype.

The majority of current research indicates that total fatty acid levels are significantly increased during tumor cell migration, invasion and metastasis. Polyunsaturated fatty acids maintain the liquidity of the cell membrane and the activa- 
tion of signaling molecules associated with cell proliferation, survival and migration. De novo activation of endogenous fatty acid synthesis may lead to changes in the FFA spectrum in the tumor cell microenvironment (34). Therefore, upon FASN inhibition, the levels of unsaturated and saturated fatty acids decreased, and cell migration was inhibited, indicating that FASN may be involved in the occurrence and development of tumors by regulating the levels of specific fatty acids. In the present study, the SKBR-3 was used as the cell line as it represents the HER-2 enriched (ER/PR negative, HER-2 positive) breast cancer subtype and the relationship between FASN and fatty acids could be investigated. Unfortunately, the number of IDC models (ER/PR-negative, HER-2-positive) in this study is small, which cannot explain the correlation between FASN and fatty acids in HER-2 rich IDC. Similarly, the present study could not explain whether hormone receptors are involved in FASN mediated fatty acids promoting breast cancer growth, so the correlation between FASN and fatty acids in luminal B (ER/PR/HER-2 positive) IDC model remains to be explained. These issues may be addressed in future experiments.

In summary, the present study determined and characterized the differences in the fatty acid profiles between patients with IDC and healthy controls, and these differences in fatty acid profiles may be attributed to variations in FASN expression levels. Following inhibition of FASN, the cell content of unsaturated and saturated fatty acids decreased and cell migration was inhibited, which indicates that a variety of fatty acids may participate in the metastasis and invasion of breast cancer cells. Changes in the expression levels of FASN in tumor cells may lead to marked changes in other metabolic enzymes and FFA activity, thereby profoundly affecting cell phenotype.

\section{Acknowledgements}

Not applicable.

\section{Funding}

The present study was supported by the Sichuan Provincial Science and Technology Department (grant no. 2016JY143).

\section{Availability of data and materials}

The datasets used and/or analyzed during the present study are available from the corresponding author on reasonable request.

\section{Authors' contributions}

HL conceived and designed the study, and also reviewed and edited the manuscript. SX performed the experiments and wrote the manuscript. TL and LD performed the data analysis. SX, TC, HW, XD, BG and HX performed the experiments. All the authors have read and approved the final manuscript.

\section{Ethics approval and consent to participate}

All patients provided written informed consent and the study was approved by the Committee on Clinical Trials and Biomedical Ethics, West China Hospital, Sichuan University.

\section{Patient consent for publication}

Not applicable.

\section{Competing interests}

The authors declare that they have no competing interests.

\section{References}

1. Ferlay J, Shin HR, Bray F, Forman D, Mathers C and Parkin DM: Estimates of worldwide burden of cancer in 2008: GLOBOCAN 2008. Int J Cancer 127: 2893-2917, 2010.

2. Global Burden of Disease Cancer C, Fitzmaurice C, Allen C, et al: Global, regional, and national cancer incidence, mortality, years of life lost, years lived with disability, and disabilityadjusted life-years for 32 cancer groups, 1990 to 2015: A systematic analysis for the global burden of disease study. JAMA Oncol 3: 524-548, 2017.

3. Cuyàs E, Corominas-Faja $\mathrm{B}$ and Menendez JA: The nutritional phenome of EMT-induced cancer stem-like cells. Oncotarget 5: 3970-3982, 2014.

4. Kuhajda FP: Fatty-acid synthase and human cancer: New perspectives on its role in tumor biology. Nutrition 16: 202-208, 2000.

5. Smith S: The animal fatty acid synthase: One gene, one polypeptide, seven enzymes. FASEB J 8: 1248-1259, 1994.

6. Hopperton KE, Duncan RE, Bazinet RP and Archer MC: Fatty acid synthase plays a role in cancer metabolism beyond providing fatty acids for phospholipid synthesis or sustaining elevations in glycolytic activity. Exp Cell Res 320: 302-310, 2014.

7. Li P, Tian W and Ma X: Alpha-mangostin inhibits intracellular fatty acid synthase and induces apoptosis in breast cancer cells. Mol Cancer 13: 138, 2014.

8. Agostini M, Almeida LY, Bastos DC, Ortega RM, Moreira FS, Seguin F, Zecchin KG, Raposo HF, Oliveira HC, Amoêdo ND, et al: The fatty acid synthase inhibitor orlistat reduces the growth and metastasis of orthotopic tongue oral squamous cell carcinomas. Mol Cancer Ther 13: 585-595, 2014.

9. De Schrijver E, Brusselmans K, Heyns W, Verhoeven G and Swinnen JV: RNA interference-mediated silencing of the fatty acid synthase gene attenuates growth and induces morphological changes and apoptosis of LNCaP prostate cancer cells. Cancer Res 63: 3799-3804, 2003.

10. Grube S, Dünisch P, Freitag D, Klausnitzer M, Sakr Y, Walter J, Kalff $\mathrm{R}$ and Ewald C: Overexpression of fatty acid synthase in human gliomas correlates with the WHO tumor grade and inhibition with Orlistat reduces cell viability and triggers apoptosis. J Neurooncol 118: 277-287, 2014.

11. Veigel D, Wagner R, Stübiger G, Wuczkowski M, Filipits M, Horvat R, Benhamú B, López-Rodríguez ML, Leisser A, Valent $\mathrm{P}$, et al: Fatty acid synthase is a metabolic marker of cell proliferation rather than malignancy in ovarian cancer and its precursor cells. Int J Cancer 136: 2078-2090, 2015.

12. Menendez JA and Lupu R: Fatty acid synthase and the lipogenic phenotype in cancer pathogenesis. Nat Rev Cancer 7: 763-777, 2007.

13. Epstein JI, Carmichael M and Partin AW: OA-519 (fatty acid synthase) as an independent predictor of pathologic state in adenocarcinoma of the prostate. Urology 45: 81-86, 1995.

14. Khan A, Aljarbou AN, Aldebasi YH, Faisal SM and Khan MA: Resveratrol suppresses the proliferation of breast cancer cells by inhibiting fatty acid synthase signaling pathway. Cancer Epidemiol 38: 765-772, 2014.

15. Zaytseva YY, Rychahou PG, Gulhati P, Elliott VA, Mustain WC, O'Connor K, Morris AJ, Sunkara M, Weiss HL, Lee EY, et al: Inhibition of fatty acid synthase attenuates CD44-associated signaling and reduces metastasis in colorectal cancer. Cancer Res 72: 1504-1517, 2012.

16. Yoon S, Lee MY, Park SW, Moon JS, Koh YK, Ahn YH, Park BW and Kim KS: Up-regulation of acetyl-CoA carboxylase alpha and fatty acid synthase by human epidermal growth factor receptor 2 at the translational level in breast cancer cells. J Biol Chem 282: 26122-26131, 2007.

17. Camp RL, Dolled-Filhart M and Rimm DL: X-tile: A new bioinformatics tool for biomarker assessment and outcome-based cut-point optimization. Clin Cancer Res 10: 7252-7259, 2004. 
18. Yu W, Chai H, Li Y, Zhao H, Xie X, Zheng H, Wang C, Wang X, Yang G, Cai X, et al: Increased expression of CYP4Z1 promotes tumor angiogenesis and growth in human breast cancer. Toxicol Appl Pharmacol 264: 73-83, 2012.

19. Maina EG, Madivoli ES, Ouma JA, Ogilo JK and Kenya JM: Evaluation of nutritional value of Asystasia mysorensis and Sesamum angustifolia and their potential contribution to human health. Food Sci Nutr 7: 2176-2185, 2019.

20. Sun L, Yao Y, Pan G, Zhan S, Shi W, Lu T, Yuan J, Tian K, Jiang L, Song S, et al: Small interfering RNA-mediated knockdown of fatty acid synthase attenuates the proliferation and metastasis of human gastric cancer cells via the mTOR/Gli1 signaling pathway. Oncol Lett 16: 594-602, 2018.

21. Wang X, Tian J, Zhao Q, Yang N, Ying P, Peng X, Zou D, Zhu Y, Zhong R, Gao Y, et al: Functional characterization of a lowfrequency V1937I variant in FASN associated with susceptibility to esophageal squamous cell carcinoma. Arch Toxicol 94: 20392046, 2020.

22. Mashima T, Seimiya $H$ and Tsuruo T: De novo fatty-acid synthesis and related pathways as molecular targets for cancer therapy. Br J Cancer 100: 1369-1372, 2009.

23. Chen T, Zhou L, Li H, Tian Y, Li J, Dong L, Zhao Y and Wei D: Fatty acid synthase affects expression of ErbB receptors in epithelial to mesenchymal transition of breast cancer cells and invasive ductal carcinoma. Oncol Lett 14: 5934-5946, 2017.

24. Shen J, Yan L, Liu S, Ambrosone CB and Zhao H: Plasma metabolomic profiles in breast cancer patients and healthy controls: By race and tumor receptor subtypes. Transl Oncol 6: 757-765, 2013

25. Lv W and Yang T: Identification of possible biomarkers for breast cancer from free fatty acid profiles determined by GC-MS and multivariate statistical analysis. Clin Biochem 45: 127-133, 2012.

26. Mohammadzadeh F, Mosayebi G, Montazeri V, Darabi M, Fayezi S, Shaaker M, Rahmati M, Baradaran B, Mehdizadeh A and Darabi M: Fatty acid composition of tissue cultured breast carcinoma and the effect of stearoyl-CoA desaturase 1 inhibition. J Breast Cancer 17: 136-142, 2014.

27. Calligaris D, Caragacianu D, Liu X, Norton I, Thompson CJ, Richardson AL, Golshan M, Easterling ML, Santagata S, Dillon DA, et al: Application of desorption electrospray ionization mass spectrometry imaging in breast cancer margin analysis. Proc Natl Acad Sci USA 111: 15184-15189, 2014.
28. Conceição LL, Dias MM, Pessoa MC, Pena GD, Mendes MC, Neves CV, Hermsdorff HH, Freitas RN and Peluzio MD: Difference in fatty acids composition of breast adipose tissue in women with breast cancer and benign breast disease. Nutr Hosp 33: 1354-1360, 2016

29. Binker-Cosen MJ, Richards D, Oliver B, Gaisano HY, Binker MG and Cosen-Binker LI: Palmitic acid increases invasiveness of pancreatic cancer cells AsPC-1 through TLR4/ROS/NF- $\kappa \mathrm{B} /$ MMP-9 signaling pathway. Biochem Biophys Res Commun 484: 152-158, 2017.

30. Röhrig F and Schulze A: The multifaceted roles of fatty acid synthesis in cancer. Nat Rev Cancer 16: 732-749, 2016.

31. Hilvo M, Denkert C, Lehtinen L, Müller B, Brockmöller S, Seppänen-Laakso T, Budczies J, Bucher E, Yetukuri L, Castillo $\mathrm{S}$, et al: Novel theranostic opportunities offered by characterization of altered membrane lipid metabolism in breast cancer progression. Cancer Res 71: 3236-3245, 2011.

32. Rysman E, Brusselmans K, Scheys K, Timmermans L, Derua R, Munck S, Van Veldhoven PP, Waltregny D, Daniëls VW, Machiels J, et al: De novo lipogenesis protects cancer cells from free radicals and chemotherapeutics by promoting membrane lipid saturation. Cancer Res 70: 8117-8126, 2010.

33. Li J, Dong L, Wei D, Wang X, Zhang S and Li H: Fatty acid synthase mediates the epithelial-mesenchymal transition of breast cancer cells. Int J Biol Sci 10: 171-180, 2014.

34. Guo S, Wang Y, Zhou D and Li Z: Significantly increased monounsaturated lipids relative to polyunsaturated lipids in six types of cancer microenvironment are observed by mass spectrometry imaging. Sci Rep 4: 5959, 2014.

(7)(3) This work is licensed under a Creative Commons CC. Attribution-NonCommercial-NoDerivatives 4.0 International (CC BY-NC-ND 4.0) License. 